\title{
Utilizing ICN/CCN for service and VM migration support in virtualized LTE systems
}

\author{
Morteza Karimzadeh, Triadimas Satria, Georgios Karagiannis \\ Department of Computer Science and the Electrical Engineering, University of Twente, Enschede, The Netherlands \\ m.karimzadeh@utwente.nl,t.a.satria@student.utwente.nl,g.karagiannis@utwente.nl
}

Keywords: Virtualized LTE, service migration, VM migration, Service Continuity, ICN/CCN.

Abstract: $\quad$ One of the most important concepts used in mobile networks, like LTE (Long Term Evolution) is service continuity. A mobile user moving from one network to another network should not lose an on-going service. In cloud-based (virtualized) LTE systems, services are hosted on Virtual Machines (VMs) that can be moved and migrated across multiple networks to such locations where these services can be well delivered to mobile users. The migration of the (1) VMs and (2) the services running on such VMs, should happen in such a way that the disruption of an on-going service is minimized. In this paper we argue that a technology that can efficiently be used for supporting service and VM migration is the ICN/CCN (Information Centric Networking / Content Centric Networking) technology.

\section{INTRODUCTION}

Long Term Evolution (LTE) is the fourth generation (4G) technology, which is standardized by the $3 \mathrm{rd}$ Generation Partnership Project (3GPP). It is capable of providing high data rates as well as support of high-speed mobility. LTE features low latency in both the control plane and the user plane, which creates new opportunities for real-time applications such as video surveillance, telemedicine, and distance learning. In the LTE system two main network parts can be identified which are called Evolved UMTS Terrestrial Radio Access Network $(e-U T R A N)$ and the Evolved Packet Core (EPC). The e-UTRAN consists of base stations denoted as Evolved Node-Bs (eNodeBs). The EPC is composed of several network elements. The main important ones are the Serving Gateway $(S-G W)$, the Packet Data Network Gateway $(P-G W)$ and the Mobility Management Entity $(M M E)$. The P-GW, that is the main mobility EPC anchor point, connects the EPC to other external networks. The S-GW supports the transport of the user data between the User Equipment $(U E)$ and the external networks. The MME is the control node that processes the mobility management signalling (i.e. handover) between the $U E$ and the EPC. Even though LTE promises a faster and more efficient data network, its architecture is still highly centralized, thus it may lead to very high bandwidth requirements on core network equipments. Long communication paths between users and servers can increase delay and waste network resources. The Mobile Cloud Networking $(M C N)$ project (EU FP7 MCN, 2013), as one of the EU FP7 projects, integrates the use of cloud computing concepts in LTE mobile networks. This is accomplished with the objective of increasing LTE's performance by building a shared distributed LTE mobile network that can: (1) optimize the utilization of computation, storage and networking resources, (2) minimize communication delays, (3) avoid bottlenecks, and (4) enable multiple network operators to create their own virtual network depending on their requirements and goals, while using a common physical infrastructure. The integration of cloud computing concepts in an LTE system, see (EU FP7 MCN, 2013), can be realized by: (1) extending the cloud computing concept beyond the typical (macro) data centers towards new smaller (micro) data centers that are distributed within the Radio Access Network (e.g., e-UTRAN) and the Mobile Core Network (e.g., EPC), see Figure 1, and (2) deploying and running cloud-based (virtualized) Radio Access Networks, denoted as RAN as a Service (RANaaS), and Mobile Core Networks, defined as EPC as a Service (EPCaaS). This trend is also in line with the emerging ETSI activities in Network Functions Virtualization $(N F V)$.

Service continuity is a critical issue in mobile networks implying access to the requested services 
without disruption, while the user moves from one network to another.

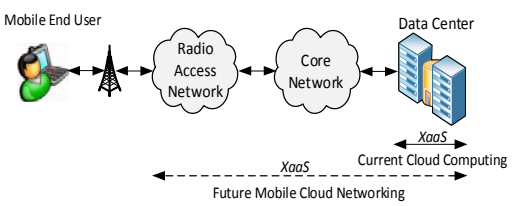

Figure 1. A view of future LTE mobile cellular network

As illustrated in Figure 2, in cloud based mobile cellular network systems services are hosted on VMs that may migrate across multiple physical networks (i.e. datacentres) with the aim of better service delivery. A service could be a content delivery or content generation and manipulation service. The migration of VMs and the services running on such VMs should occur in such a way that the disruption of an on-going service is minimized.

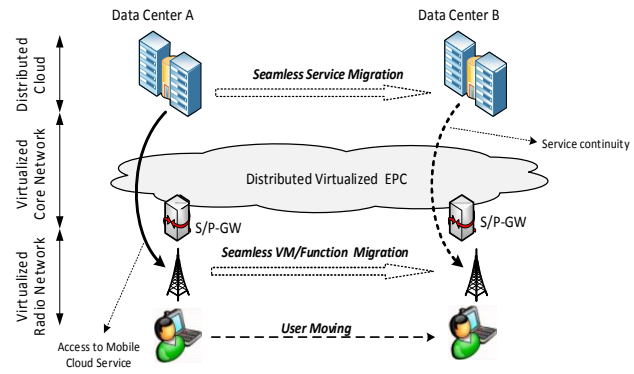

Figure 2. Service continuity in the virtualized LTE system

A service continuity solution should be able to support the migration of services, implying the support for:

- IP address continuity: When a user moves to another sub-network, the application will not observe the change of the IP address.

- Session continuity: It is a combination of IP address continuity and service context migration. Service context migration occurs when a user moves to a new location and the service context used by the function in the previous location should be able to be migrated and used by the same function at the new location.

- Content continuity: Refers to migration and delivery of the requested content from a location close to the mobile user. The requested content can be migrated and delivered from a location close to a mobile user.

- Storage continuity: Storage should be able to migrate to a new location close to the mobile user
- Function continuity: The same function in a new location can be run using context used by the same function in the previous location. Function migration needs to be supported in order to maintain function continuity.

Several technologies can be considered as possible candidates for the support of service and VM migration in virtualized LTE systems. For example, live VM migration solutions have been proposed in the literature, see e.g., (Mandal et al, 2013), but none of these can be used efficiently to support the service continuity requirements listed above.

Moreover, in this paper we argue that the best candidate technology that can efficiently be used for the support of service and VM migration in virtualized LTE systems is the Information Centric Networking/Content Centric Networking (ICN/ $C C N)$. In particular, this paper answers the following research question:

"Can the ICN/CCN technology be used efficiently for the support of service and VM migration in the virtualized LTE?"

This paper is organized as follows. Section 2 provides an overview of the ICN/CCN concept and explains how it could be exploited to support service continuity in virtualized LTE systems. Section 3, briefly introduces other possible candidate technologies and compares them with the ICN/CCN approach. Section 4 explains by using an example how the ICN/CCN concept can be applied in the virtualized LTE system. Moreover, Sections 3 and 4 are answering the research question listed above. Finally, Section 5 concludes the article and provides recommendations for future work.

\section{2. $\mathrm{ICN} / \mathrm{CCN}$}

ICN is an Internet architecture approach based on Named Data Objects (NDOs), see e.g., (Ahlgren et al, 2012), (Pentikousis et al, 2013). It changes the focal point of the network architecture from the "end host" to "information" (content or data). The ICN architecture leverages in-network storage for caching, multiparty communication through replication, and interaction models decoupling senders and receivers (Koponen et al, 2007). The NDO, such as web page, video, document, or other kind of information, is independent of the location, the storage method, the application program, and the transportation method. A unique name for each 
$\mathrm{NDO}$ is required in ICN in order to identify objects independently of their locations. Information about the source of an object is also useful to associate with the name. ICN has an API, which is used, as an interface for publishing or getting NDOs. By using the ICN API, a producer can publish NDOs to the Internet and a consumer can get them from internet.

Advantages of the ICN approach could be summarized as follows (Koponen et al, 2007) :

- The ICN approach offers scalable and costeffective content distribution as it leverages innetwork caching, so that requests for NDOs can be served by any network node holding a copy of the requested NDOs.

- It benefits from persistent and unique naming of NDOs, and also a service model that decouples senders and receivers, so that it does not have a problem with name-object binding.

- The ICN approach has an interesting security model. It provides name-data integrity and original verification of NDOs, independently of the immediate source. Hence, it enables ubiquitous caching with retained name-data integrity and authenticity.

- The ICN approach supports IP address continuity and multi-homing. A mobile client just needs to send requests for NDOs to a new access and the requests will be served by a network node that might be different from the previous network node.

- The ICN approach provides better reliability and performance compared to the current networks since it leverages optimized hop-by-hop transport and in-network caching.

Currently there are several ICN approaches that have been developed, such as Data-Oriented Network Architecture (DONA) (Koponen et al, 2007), Content-Centric Networking (CCN) (Jacobson et al, 2009), Publish-Subscribe Internet Routing Paradigm (PSIRP) (Fotiou et al, 2012), Network of Information (NetInf) (Dannewitz et al, 2013) and Translating Relaying Internet Architecture integrating Active Directories (TRIAD) (Gritter and Cheriton, 2000). These ICN approaches undergo the lack of efficient support of session and function continuity. However, the Service-Centric Networking (SCN) (Braun et al, 2011) concept, which is a new ICN based concept is able to support session and function continuity. SCN is a new networking paradigm for the future Internet, in which routing and forwarding are based on service identifiers. SCN is an extension of CCN, see Section 2.1, which is designed based on an object-oriented approach, in which the contents and the services are considered as objects. In SCN, the content not only can be retrieved but also can be processed before being delivered to users. In SCN, services are represented as functions to be invoked by users. By using an object-oriented approach, both functions and data are integrated into objects. In SCN, clients can request for both services and contents by using object names.

\subsection{CCN Concept}

The transport protocol used in CCN (Jacobson et al, 2009) is called CCNx that is used to distribute information related to the location of NDOs published on nodes. There are two types of $\mathrm{CCNx}$ messages, namely the Interest message, which contains the request for an NDO and the Data message, which contains the response for an Interest message.

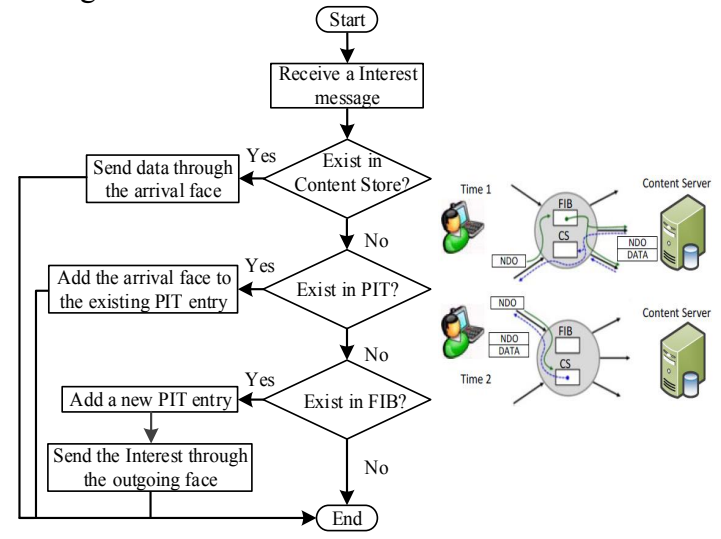

a. Interest message processing $\quad b$. Data forwarding

Figure 3. $\mathrm{CCN}$ protocol Overview

The CCNx protocol, see Figure 3, operates based on three main data structures as follows:

- Content Store (CS): CS represents a buffer memory used for data retrieval by prefix match lookup on names.

- Forwarding Information Base (FIB): FIB contains a list of entries with interfaces to where the Interest messages should be forwarded. Each entry in the FIB may point to multiple interfaces to where the Interest messages could be forwarded.

- Pending Interest Table (PIT): PIT is used to keep track of Interest messages forwarded upstream. It contains information of sources of unsatisfied Interests. Each entry in the PIT may point to multiple sources. 


\section{MOTIVATION: WHY ICN/CCN SHOULD BE USED FOR SERVICE AND VM MIGRATION SUPPORT IN VIRTUALIZED LTE SYSTEMS}

This section introduces other possible candidate technologies for service and VM migration support and analyses and compares them with the ICN/CCN approach in order to verify whether they can be applied in a virtualised LTE system.

\subsection{Mobile Content Distribution Networks (M-CDN)}

The deployment and distribution of cache servers in the Internet, enables the distribution of load in serving the increasing number of requests of content, such as web content, video content or software.

Current caching products can be deployed solely outside a mobile operator's network, such as the solutions offered by Akamai (Nygren et al, 2010), or close to/at the mobile operator's gateways that support mobility anchor functionality. The aim of M-CDN, see e.g., (Saroiu et al, 2002), is to integrate cache servers with the mobile operator's core network or even with its access and backhaul network in order to move these cache servers, as content sources, closer to mobile users.

\subsection{Software-Defined Networking (SDN)}

Open Networking Foundation $(O N F)$ defined SDN as an emerging network architecture where network control is decoupled from forwarding and is directly programmable (ONF, April 2012). The SDN architecture supports network virtualization since the underlying network infrastructure can be abstracted from the applications and network services. It provides a new dynamic network architecture, which changes traditional network platforms into rich service-delivery platforms. In practice, SDN refers more broadly to logically centralized software control. The network appears to the applications and policy engines as a single logical switch since the network intelligence that maintains a global view of the network is logically centralized in softwarebased SDN controllers.

\subsection{Host Identity Protocol (HIP)}

HIP introduces a new layer between the network and transport layers, which maps the host identifiers to network addresses and vice versa (Moskowitz and Nikander, 2006). In the HIP architecture, the endpoint names and locators, which are both represented by IP addresses in the current Internet architecture, are separated. IP addresses will act as locators, while the host identifiers take the role of end-point identifiers. The existence of Host Identity Layer ensures that the transport layer connections are no more bound to IP addresses, so that if the location of a host changes, the connections will not have to be broken.

\subsection{SCTP with dynamic address reconfiguration extension}

T. Dreibholz et.al in (Dreibholz et al, 2003) proposed a new scheme for mobility management for IP-based networks relying on a transport protocol called Stream Control Transmission Protocol (SCTP), with the extension for dynamic address reconfiguration, and the Reliable Server Pooling (RSerPool) protocol suite. The proposed solution is transparent to application, and does not require changes in the network infrastructure. SCTP, specified in IETF RFC 4960, is a transport protocol that provides a more flexible data delivery by separating reliable transfer of messages between endpoints from the actual delivery to the user process. It supports multi-homed endpoints with more than one IP address, so that it provides an improved network-level fault tolerance.

\subsection{Proxy Mobile IPv6 (PMIPv6)}

PMIPv6 is a network-based mobility management protocol (Gundavelli et al, 2008) based on the Mobile IPv6 concept, specified in IETF RFC 3775, which is designed to provide mobility management support to a mobile node without requiring participation of the mobile node in any IP mobility related signalling. There are two core functional entities in PMIPv6 used to track movements of mobile nodes and initiate the mobility signalling and set up the required routing state, namely Local Mobility Anchor (LMA) and Mobile Access Gateway $(M A G)$. The LMA is the topological anchor point for the mobile node's home network prefixes used to maintain a reachability state of the mobile node, while the MAG is the entity used to 
perform mobility management for the node attached to its access link.

\subsection{Identifier-Locator Network Protocol (ILNP)}

Randall Atkinson et.al, in (Atkinson et al, 2009) proposed the ILNP, a naming architecture which addresses issues of mobility, multi-homing, and endto-end IP security. It evolves the naming in the Internet by splitting an address into two different entities, as Identifier $(I)$ used for end-to-end identity and Locator $(L)$ used for routing and forwarding packets. It provides an integrated solution to the issues mentioned above without changing the core routing architecture, while offering incremental deploy-ability through backward compatibility with IPv6.

\subsection{Analysis and comparison}

To support service continuity in virtualised LTE systems, the selected technology needs to support requirements, such as IP address continuity, session continuity, content continuity, storage continuity and function continuity, see Section 1. Table 1 analyses and compares ICN/CCN with other candidate technologies that were briefly described in the previous subsections, taking into account the requirements listed in Section 1.

Table 1. The list of solutions and their ability in supporting service continuity (S: Supported, NS: Not Supported).

\begin{tabular}{|c|c|c|c|c|c|}
\hline 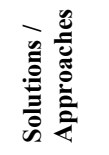 & 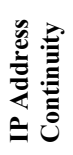 & 总 & 总 & 总 & 氞 \\
\hline ICN & $S$ & $S$ & $S$ & $S$ & $S$ \\
\hline M-CDN & $\mathrm{S}$ & $\mathrm{S}$ & $\mathrm{S}$ & $\mathrm{S}$ & NS \\
\hline SDN & $\mathrm{S}$ & $\mathrm{S}$ & $\mathrm{S}$ & $\mathrm{S}$ & $\mathrm{S}$ \\
\hline HIP & $\mathrm{S}$ & NS & NS & NS & NS \\
\hline SCTP & $S$ & NS & NS & NS & NS \\
\hline PMIPv6 & $\mathrm{S}$ & NS & NS & NS & NS \\
\hline ILNP & $\mathrm{S}$ & NS & NS & NS & NS \\
\hline
\end{tabular}

Based on the analysis and comparison given in Table 1, it can be deduced that the ICN/CCN and SDN solutions can satisfy all requirements for service continuity. This also implies that the ICN/CCN technology is a promising candidate that can efficiently be used for the support of service and VM migration in the virtualized LTE system. The proposed ICN/CCN approach could also offer reliable, scalable and cost-effective content distribution by leveraging in-network caching. This solution may be integrated with the SDN approach, by utilizing OpenFlow switches in order to provide a dynamic network architecture that fosters network virtualization and can improve network scalability, manageability and agility. The centralized network state in the control layer provides simplicity and flexibility for network designers and network operators to configure and improve their networks using automated SDN programs. However, the deployment of SDN requires significant changes on the existing network infrastructure.

\section{EXAMPLE OF INTEGRATING ICN/CCN IN A VIRTUALIZED LTE SYSTEM}

This section provides an example on how the ICN/CCN concept can be applied in a virtualized LTE system. As shown in

Figure 4, the assumption in this architecture is that the eNodeBs, S-GWs and P-GWs are CCN capable (i.e., able to support the CCNx protocol) and that the routers deployed in the infrastructure used to interconnect the RAN and EPC components are non CCN capable.

Furthermore, it is also considered that the Content Delivery Network $(C D N)$ engine/ repository is $\mathrm{CCN}$ capable and could provide the requested content/data to the $\mathrm{CCN}$ infrastructure faster than the main server that stores the content. Utilizing the CDN repository could effectively accelerate the content delivery to users and reduce bandwidth consumption in the network.

In this figure Virtualization Controlling Platform $(V C P)$ represents the cloud components, which carry out the signalling and configuration of the virtualized LTE elements (e.g., eNodeBs, S-GWs and P-GWs) in order to support service continuity, see (EU FP7 MCN, 2013).

It is also considered that UEs are $\mathrm{CCN}$ capable, meaning that they are able to generate, send and receive the Interest and Data messages.

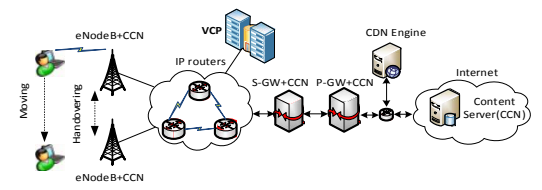

Figure 4. Integrating ICN/CCN in LTE system 
When a UE that uses both a service and a virtualized $\mathrm{CCN}$ process hosted on a (source) data centre, moves towards another (destination) data centre hostsing another virtualised $\mathrm{CCN}$ process, the ICN/CCN approach can be used to migrate the ongoing service from the source data centre to the new destination data centre. The ICN/CCN approach can also be used to migrate a VM running on the source data centre to the new destination data centre. The latter can be accomplished by enabling the destination data centre to request an on-going VM from the source data centre, by using one or more Interest messages.

\section{CONCLUSIONS AND FUTURE WORK}

By analyzing and comparing different technologies, this paper argued and verified that the ICN/CCN technology is a promising candidate that can efficiently be used for the support of service and VM migration in the virtualized LTE system.

In order to further validate this statement, the use of the ICN/CCN technology for the support of service and VM migration in the virtualized LTE system will be prototyped and evaluated within the context of the EU FP7 project (EU FP7 MCN, 2013).

\section{ACKNOWLEDGEMENTS}

This work is accomplished in the context of the EU FP7 Mobile Cloud Networking $(M C N)$ project. Therefore, we would like to acknowledge the European Commission, since the MCN project is an EC funded Integrated Project under the 7th RTD Framework Programme, FP7-ICT-2011-8-grant agreement number 318109 .

\section{REFERENCES}

EU FP7 MCN, (visited on September 2013), $<$ http://www.mobile-cloud-networking.eu/site/>.

Mandal, U., Habib, M., Shuqiang , Z., Mukherjee, B., Tornatore, M., 2013. Greening the cloud using renewableenergy-aware service migration, IEEE Network, vol. 27, Iss. 6. 36-43, IEEE.

Ahlgren, B., Dannewitz, C., Kutscher, D., Ohlman, B., July 2012. A survey of information-centric networking. In IEEE Communication Magazine vol. 50, pp. 26-36. IEEE.
Pentikousis, K., Ohlman, B., Corujo, D., Boggia, G., Tyson, G. Davies, E., Mahadevan, P., Spirou, S., Molinaro, A., Gellert, D., Eum, S., July 2013. ICN Baseline Scenarios and Evaluation Methodology. IETF Internet draft (work in progress), IETF.

Koponen, T., Chawla, M., Chun, B.-G., 2007. A DataOriented (and Beyond) Network Architecture. In Proc. of ACM SIGCOMM '07, vol. 37, Iss. 3, pp. 181-192, ACM.

Jacobson, V., Smetters, D., Thornton, J., Plass, M., Briggs, N., Braynard, R., 2009. Networking named content. In Proc. of 5th ACM International Conference on Emerging Networking Experiments and Technologies (CoNEXT'09), ACM.

Fotiou, N., Trossen, D., Polyzos, G.C., 2012. Illustrating a publish-subscribe Internet architecture. Journal of Telecommunication Systems, Vol. 51, Iss. 4, pp. 233-245, Springer.

Dannewitz, C., Kutscher, D., Ohlman, B., Farrell, S., Ahlgrene, B., Karla, H., 2013. Network of Information (NetInf) - An information-centric networking architecture. Journal of Computer Communications, Vol. 36, Iss. 7, pp. 721-735, Elsevier.

Gritter, M., Cheriton, D.R., Jan 2000. TRIAD: A New Next-Generation Internet Architecture, (visited in December 2013) < http://www-dsg.stanford. edu/triad/>.

Braun, T., Hilt, V., Hofmann, M., Rimac, I., Steiner, M., Varvello, M., 2011. Service-Centric Networking. In Proc. of 4th International Workshop on the Network of the Future.

Nygren, E., Sitaraman, R.K., Jennifer, S., 2010. The Akamai network: a platform for high-performance Internet applications. ACM SIGOPS Operating Systems Review, Vol. 44, No.3, ACM.

Saroiu, S., Gummadi, K.P., Dunn, R.J., Gribble, S.D., Levy, H.M., 2002. An Analysis of Internet Content Delivery Systems. In Proc. of OSDI '02.

ONF, April 2012. White paper,. Open Networking Foundation, (visited in December 2013)

$<$ https://www.Opennetworking.org/>.

Moskowitz, R., Nikander, P., 2006. Host Identity Protocol (HIP) Architecture. IETF RFC4423, IETF.

Dreibholz, T., Jungmaier, A., Tuxen, M., 2003. A New Scheme for IP-based Internet-Mobility. In Proc. of the 28th Annual IEEE International Conference on Local Computer Networks (LCN '03), IEEE.

Gundavelli, S., Leung, K., Devarapalli, V., Chowdhury, K., August 2008, Proxy Mobile IPv6, IETF RFC 5213, IETF.

Atkinson, R., Bhatti, S., Hailes, S., 2009. ILNP: Mobility, Multi-Homing, Localised Addressing and Security Through Naming. Telecommunication Systems, vol. 42, no. 3-4 pp. 273-291, Springer. 